\title{
Segregation in inclined flows of binary mixtures of spheres
}

\author{
Michele Larcher ${ }^{1, *}$, and James T. Jenkins ${ }^{2}$ \\ ${ }^{1}$ Faculty of Science and Technology, Free University of Bozen-Bolzano, Bozen-Bolzano, 39100, Italy \\ ${ }^{2}$ School of Civil and Environmental Engineering, Cornell University, Ithaca, NY, 14853, USA
}

\begin{abstract}
We outline the equations that govern the evolution of segregation of a binary mixture of spheres in flows down inclines. These equations result from the mass and momentum balances of a kinetic theory for dense flows of inelastic spheres that interact through collisions. The theory employed for segregation is appropriate for particles with relatively small differences in size and mass. The flow of the mixture is assumed to reach a fully developed state much more rapidly than does the concentrations of the two species. We illustrate the predictions of the theory for a mixture of spheres of the same diameter but different masses and for spheres of different diameters but nearly the same mass. We show the evolution of the profiles of the concentration fractions of the two types of spheres and the profiles in the final, steady state. The latter compare favourably with those obtained in discrete-element numerical simulations.
\end{abstract}

\section{Introduction}

Larcher \& Jenkins [1] predict the evolution of concentration profiles in dry flows of a binary mixture of inelastic spheres in dense inclined flows. For the mixture, they employed the extension of kinetic theory for identical, inelastic spheres developed by Garzo \& Dufty [2], modifying the expression for energy dissipation to take in to account the formation of particle clusters [3-5]. The theory employed for segregation is appropriate for particles with relatively small differences in size and mass. In doing this, they assumed that the flow of the mixture reaches a fully developed state much more rapidly than does the concentrations of the two species. This is consistent with the hypothesis of dense flow, because the rate at which segregation takes place is proportional to the average distance between the edges of spheres, while the rate at which momentum is transferred in the mixture is inversely proportional to this distance [1].

As do Larcher \& Jenkins [1], we consider a mixture of spherical particles with two different radii, $r_{A}$ and $r_{B}$, with $r_{A B}=r_{A}+r_{B}$, masses $m_{A}$ and $m_{B}$, with $m_{A B}=m_{A}+$ $m_{B}$, number densities, $n_{A}$ and $n_{B}$, with $n=n_{A}+n_{B}$, and mass densities, $\rho_{A}=m_{A} n_{A}$ and $\rho_{B}=m_{B} n_{B}$, with $\rho=\rho_{A}+$ $\rho_{B}$. We predict the evolution of the profiles of the species concentration fraction, $c_{A} / c$, where $c_{A}=4 \pi n_{A} r_{A}^{3} / 3$ and $c=$ $c_{A}+c_{B}$, for two combinations of particle radii and particle masses in time-dependent segregation under gravity. The flow is inclined by an angle $\phi$, the gravitational acceleration is $g$, and the strength $T$ of the velocity fluctuations of the mixture is equal to one-third the mean of the square of their magnitude. We compare the steady states obtained with the profiles measured by Tripathi \& Khakhar [6] in discrete numerical simulations.

\section{Governing equations}

\subsection{Segregation}

Larcher \& Jenkins [1] characterize the segregation of two types of spheres using the measure $X=\left(n_{A}-n_{B}\right) /(2 n)$ of segregation and show that when the differences $\delta r=\left(r_{A} / r_{B}\right)-1 \quad$ and $\delta m=\left(m_{A}-m_{B}\right) / m_{A B}$, in radii and masses are small, the evolution of the segregation is uniform inclined flow is governed by

$$
\rho \frac{\partial X}{\partial t}+\frac{\partial}{\partial y}\left[\frac{m_{A} n}{4}\left(1-4 X^{2}\right)\left(\tilde{v}_{A}-\tilde{v}_{B}\right)\right]=0,
$$

where $y$ varies across the flow from 0 at the base to $h$ at the upper surface, and

$$
\begin{aligned}
& \tilde{v}_{A}-\tilde{v}_{B}=-D_{A B}\left[(6.2 \delta m-4.4 \delta r) G \frac{T^{\prime}}{T}\right. \\
& \left.-(\delta m-1.5 \delta r) \frac{m_{A B} g \cos \phi}{T}+\frac{X^{\prime}}{0.25-X^{2}}\right],
\end{aligned}
$$

with the prime denoting a $y$-derivative, gives the difference of the diffusion velocities for the two species normal to the bed; the diffusion coefficient, $D_{A B}$, is given by

$$
D_{A B} \equiv \frac{\pi^{1 / 2}}{16} \frac{r_{A B}}{G}\left(\frac{2 T}{m_{A B}}\right)^{1 / 2},
$$


in which

$$
G=5.69 c \frac{\left(c_{M}-0.49\right)}{\left(c_{M}-c\right)}
$$

is the product of the granular concentration, $c$, and the radial distribution function for dense flows [2]. The value of the singularity, $c_{M}$, in the radial distribution function is taken to be 0.58 . This provides the best fit to the simulations of Tripathi \& Khakhar [3,6].

We take $N_{A}, V_{A}, N_{B}$ and $V_{B}$ to be the total numbers and volumes of spheres of species $A$ and $B$, respectively, and $N=N_{A}+N_{B}$ and $V=V_{A}+V_{B}$. In the discrete numerical simulations, the total number fraction $N_{A} / N$ or, equivalently, the total volume fraction $V_{A} / V$ of spheres is specified. In order to incorporate this information [3], the calculation is effected in terms of the variable $\zeta \equiv X n / \bar{n}$, where

$$
X \doteq \frac{N}{\bar{c}}\left(\frac{\bar{c}_{A}}{N_{A}}+\frac{\bar{c}_{B}}{N_{B}}\right) \zeta
$$

using

$$
\bar{\zeta}=\frac{\left(\bar{n}_{A}-\bar{n}_{B}\right)}{2 \bar{n}}=\frac{\left(N_{A}-N_{B}\right)}{2 N}
$$

In these expressions, the overbar denotes depth-averaged quantities.

With equations (2-6), eq. (1) can be solved for the variable $\zeta$; then, the time evolution of the concentration profiles of the two species can be evaluated through eq. (5) and

$$
\begin{aligned}
& c_{A}=-\frac{(2 X+1)\left(r_{A} / r_{B}\right)^{3} c}{(2 X-1)-(2 X+1)\left(r_{A} / r_{B}\right)^{3}}, \\
& c_{B}=-\frac{(2 X-1) c}{(2 X-1)-(2 X+1)\left(r_{A} / r_{B}\right)^{3}} .
\end{aligned}
$$

\subsection{Mixture fields}

The mixture temperature, velocity, and concentration are determined, as outlined by Larcher \& Jenkins [3]. The profile of the granular temperature, $T$, is

$$
T=\frac{m_{A B}(h-y)}{4(1+e) G} g \cos \phi(1+2 \bar{X} \delta m),
$$

where $e$ is an effective coefficient of restitution [3], $J=$ $(1+e) / 2+(\pi / 4)(3 e-1)\left(1+e^{2}\right) /[24-(1-e)(11-e)][2]$ and

$$
\begin{aligned}
& G=\left\{\frac{4 J}{5 \pi^{1 / 2}} \frac{1}{1+e}\left[\frac{30}{J}\left(1-e^{2}\right)\right]^{1 / 3} \frac{1}{\tan \phi}\right\}^{9} \\
& \times[1+3 \bar{X}(\delta r+\delta m)] .
\end{aligned}
$$

The profile of the mixture flow velocity, $u$, is

$$
\begin{aligned}
& u=\frac{5 \pi^{1 / 2}}{6 J} \frac{1}{r_{A B}}\left(\frac{1+e}{2 G} g \cos \phi\right)^{1 / 2} \\
& \times\left[h^{3 / 2}-(h-y)^{3 / 2}\right] \tan \phi(1-\bar{X} \delta r),
\end{aligned}
$$

where the slip velocity has been neglected because it does not affect significantly the prediction of granular segregation [3].

The concentration of the mixture, $c$, can be obtained by inverting eq. (4) and making use of eq. (10):

$$
c=\frac{c_{M} G}{G+5.69\left(c_{M}-0.49\right)} .
$$

It should be noted that the mixture velocity, temperature and concentration are influenced by the size and mass differences of the two types of spheres through the parameters $\delta m$ and $\delta r$ and by their volume fraction through the depth-averaged segregation measure $\bar{X}$ :

$$
\bar{X}=\frac{1}{4}\left[\frac{V_{A}}{V}-\left(1-\frac{V_{A}}{V}\right)\left(\frac{r_{B}}{r_{A}}\right)^{3}\right]\left[1+\left(\frac{r_{B}}{r_{A}}\right)^{3}\right] .
$$

\section{Predictions}

For an inclination of $\phi=25^{\circ}$, we predict the evolution from a perfectly mixed state of the concentration fractions for two systems: spheres of equal size, but different masses (Fig. 1); and approximately equal masses, but different sizes (Fig. 2).

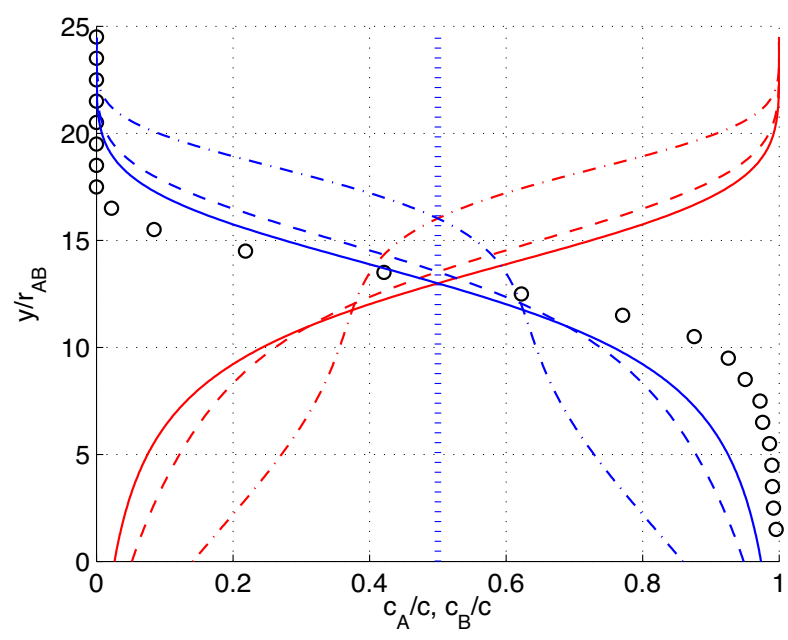

Fig. 1. Predicted profiles, $c_{A} / c$, of the more massive spheres (decreasing left to right, blue lines) and of the less massive spheres, $c_{B} / c$, (increasing left to right, red lines) at four equally spaced time intervals: $\Delta \tau=19800, r_{A} / r_{B}=1, m_{A} / m_{B}=3, V_{A} / V$ $=0.5$. The black circles indicate the steady profile of the more massive spheres in the discrete numerical simulations of Tripathi \& Khakhar [6]. The initial states (dotted lines) evolve through two subsequent intermediate states (dash-dotted and dashed lines) to the steady state (solid lines).

The curves are at equally spaced time intervals $\Delta \tau$, with time made dimensionless by $\left(r_{A B} / g\right)^{1 / 2}$, from a 
homogeneously mixed state to the final steady state through two subsequent intermediate states. The black circles indicate the steady profile of the more massive (Fig. 1) or the larger (Fig. 2) spheres in the discrete numerical simulations of Tripathi \& Khakhar [6].

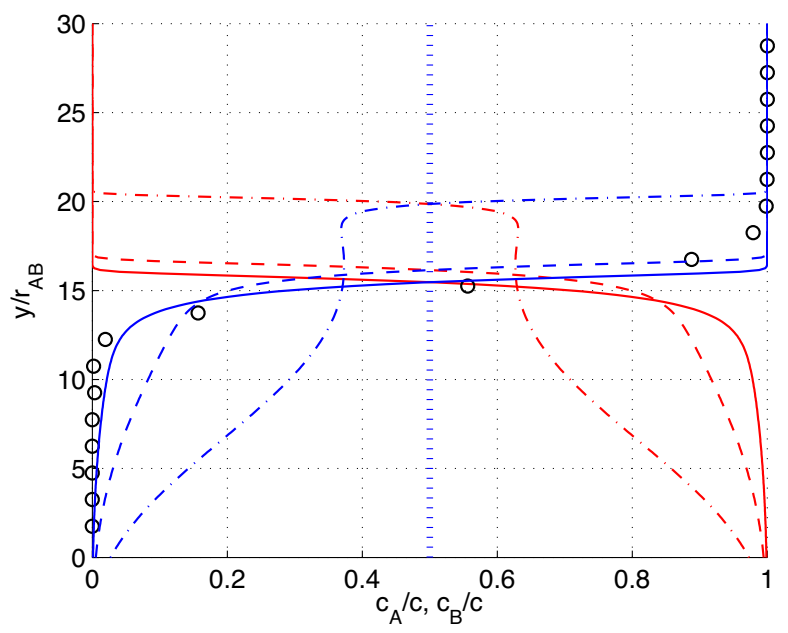

Fig. 2. Predicted profiles of $c_{A} / c$ for the larger spheres (increasing left to right, blue lines) and for the smaller spheres, $c_{B} / c$, (decreasing left to right, red lines): $\Delta \tau=4950, e=0.7$, $r_{A} / r_{B}=1.5, V_{A} / V=0.5$. The black circles indicate the steady profile of the larger spheres in the discrete numerical simulations of Tripathi \& Khakhar [6]. The initial states (dotted lines) evolve through two subsequent intermediate states (dashdotted and dashed lines) to the steady state (solid lines).

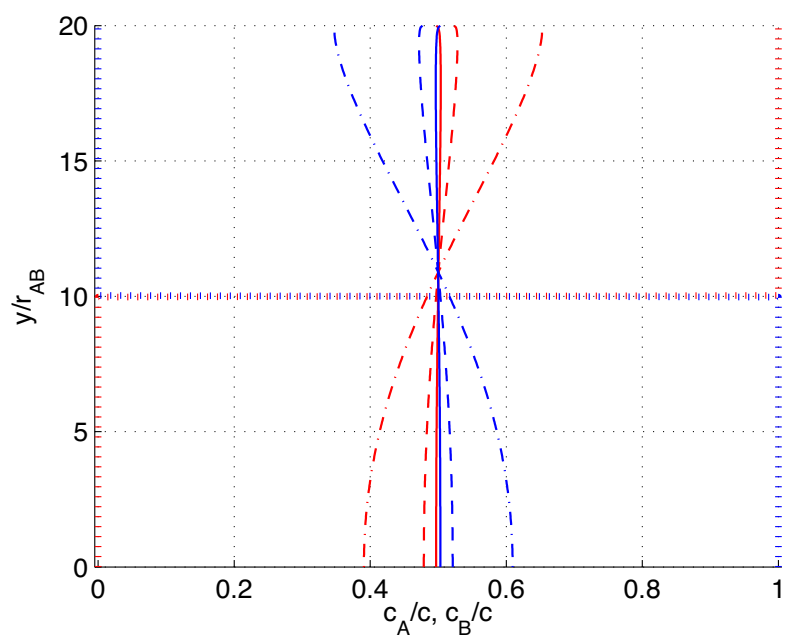

Fig. 3. Predicted profiles of $c_{A} / c$ for the large, heavy spheres (decreasing left to right, blue lines) and for the smaller spheres, $c_{B} / c$, (increasing left to right, red lines) at four equally spaced time steps from the initial state (dotted lines) to the final, mixed state (solid lines): $\Delta \tau=30000, e=0.7, r_{A} / r_{B}=1.0375, m_{A} / m_{B}$ $=2\left(r_{A} / r_{B}\right)^{3}, V_{A} / V=0.5, \phi=25^{\circ}, \mathrm{h} / r_{A B}=20$. Initially, all the large, heavy particles are in the lower half of the flow.

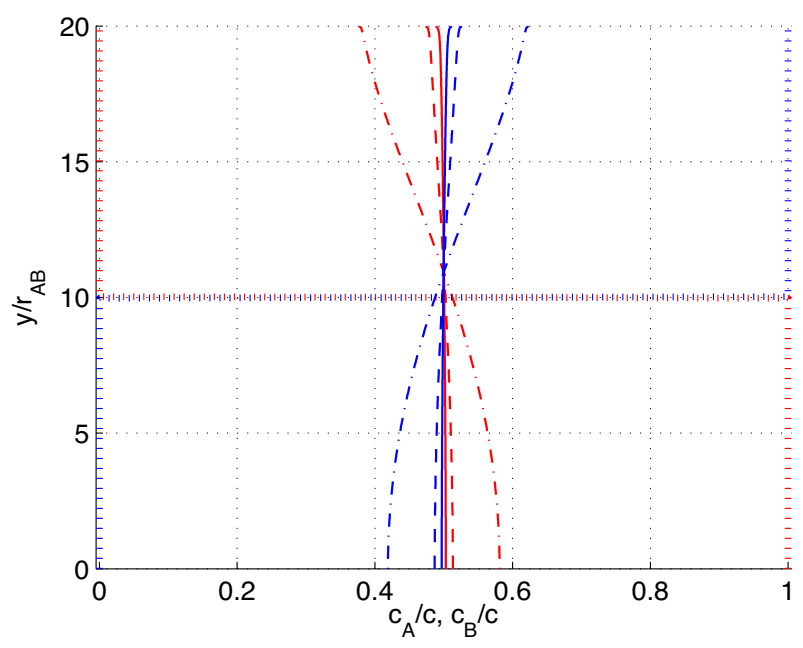

Fig. 4. Predicted profiles of $c_{A} / c$ for the large, heavy spheres (increasing left to right, blue lines) and for the smaller spheres, $c_{B} / c$, (decreasing left to right, red lines) at four equally spaced timesteps from the initial state (dotted lines) to the final, mixed state (solid lines): $\Delta \tau=36000, e=0.7, r_{A} / r_{B}=1.0375, m_{A} / m_{B}$ $=2\left(r_{A} / r_{B}\right)^{3}, V_{A} / V=0.5, \phi=25^{\circ}, \mathrm{h} / r_{A B}=20$. Initially, all the small, light spheres are in the lower half of the flow.

For bimodal mixtures of spheres with the same density but different size, the larger particles tend to concentrate at the top of the flow (Fig. 2). In the case of mixtures of species with the same size but different density (Fig. 1), the more massive spheres migrate downwards. Similar findings were shown in free surface flows [7], rotating tumblers $[8,9]$ and rotating tubes [10].

Due to the competing role of size and mass described above in the evolution of segregation, a particular mixture of large heavy and small light spheres might produce a perfect mixing. This is shown in the next two figures, where two types of spheres, completely separated initially, evolve towards a perfect mixing. The theory predicts a slightly faster evolution if the large, heavy particles are initially concentrated in the lower half of the flow (Fig. 3). In the opposite case (Fig. 4), the steady state condition is exactly the same, although it is reached in a longer time.

\section{Discussion}

We have outlined a theoretical model for the time evolution of particle segregation of dense, collisional channel flows driven by gravity in the absence of sidewalls. An extension of kinetic theory for dense, dissipative flows was used to derive the profiles of velocity, granular temperature and concentration for the mixture, while a kinetic theory for a binary mixture of nearly elastic spheres was used to describe the time evolution of the concentration profiles for the two types of spheres.

We tested the theory against the numerical simulations of Tripathi and Khakhar [6] and showed good agreement both for the case of particles of the same 
size and different mass and for particles of different size and nearly the same mass.

The theory predicts a larger influence of size than of mass on the time needed to reach a steady state: the relative concentration of particles with a size ratio of 1.5 and nearly the same mass (Fig. 2) evolves approximately four times faster than particles of the same size having a mass ratio equal to 3 (Fig. 1). The predictions of perfect mixing reinforce the fact that the size dominates mass. In this case, particles with a density that is twice as large as that of the other species, need only be about $4 \%$ larger, in order to inhibit the segregation mechanism.

\section{References}

1. M. Larcher, J.T. Jenkins, J. Fluid Mech. 782, 405429 (2015)

2. V. Garzo, J.W. Dufty, Phys. Rev. E 14, 341-365 (2004)

3. M. Larcher, J.T. Jenkins, Phys. Fluids 25, 113301 (2013)

4. J.T. Jenkins, Granul. Matt. 10, 47-52 (2007)

5. J. T. Jenkins, D. Berzi, Granul. Matt.12, 151-158 (2010)

6. A. Tripathi, D.V. Khakhar, Phys. Fluids 23, 113302 (2011)

7. J. A. Drahun, J. Bridgewater, Powder Technol. 36, 39-53 (1983)

8. G. Felix, N. Thomas, Phys. Rev. E 70, 051307 (2004)

9. K. M.Hill, Y. Fan, J. Zhang, C. V. Niekerk, E. Zastrow, S.C. Hagness, J.T. Bernhard, Granul. Matt. 12, 201-207 (2010)

10. G. Metcalfe, M. Shattuck, Physica A 233, 7097171996 (1996) 